\title{
Influencia de Brasil en la Política Alimentaria Latinoamericana: el programa de compras públicas a la agricultura familiar de Chile*
}

\author{
Influence of Brazil on Latin American \\ Food Policy: the public purchase program \\ to family farming in Chile
}

Lourrene Maffra** y Sofía Boza***

\begin{abstract}
RESUMEN
El objetivo del presente artículo es explorar la influencia brasileña sobre la política alimentaria en Latinoamérica dentro de un marco de cooperación sur-sur basado en la transferencia. Para ello se consideró el caso de los programas de compras públicas de alimentos a la agricultura familiar tanto de Brasil, pionero a nivel regional, como de Chile, donde fue implantado durante el segundo gobierno de la Presidenta Michelle Bachelet. En una primera parte se describen y analizan ambos programas. A continuación, se examina la influencia del programa brasileño en su par chileno a partir de una revisión de los mecanismos de intercambio y cooperación entre Chile y Brasil, sobre todo en materia alimentaria. Como resultado general, aunque se observan importantes diferencias en términos operativos entre ambos programas, la influencia brasileña se percibe claramente en el establecimiento de la agenda.
\end{abstract}

\footnotetext{
** Departamento de Relaciones Internacionales, Universidade Federal do Amapá (UnIfAP), Brasil. lourrenemaffra@unifap.br.

:** Departamento de Economía Agraria, Facultad de Ciencias Agronómicas e Instituto de Estudios Internacionales, Universidad de Chile. sofiaboza@u.uchile.cl.

Recibido: 9 de junio de 2018. Modificado: 26 de diciembre de 2019. Aceptado: 25 de enero de 2020.
} 
Palabras clave: Política alimentaria - compras públicas - transferencia de políticas - cooperación sur-sur - Brasil - Chile.

\section{ABSTRACT}

The objective of this article is to explore the Brazilian influence in Latin American food policy within a framework of southsouth cooperation based on policy transfer. For this, the cases of the program of public purchases from family farming in Brazil, pioneer at regional level, and Chile, initiated during the second term of president Michelle Bachelet, are considered. In the first part, both programs are described and analyzed. Next, we examine the influence of the Brazilian program on its Chilean counterpart. This based on a review of the mechanisms of exchange and cooperation between Chile and Brazil, especially in agri-food policies. As general result, although there are important differences in operational terms between both programs, the Brazilian influence is clearly perceived in the agenda setting.

Keywords: Food policy - public purchases - policy transfer south-south cooperation - Brazil - Chile. 


\section{INTRODUCCIÓN}

A partir de la década del 2000, los países en desarrollo profundizan las iniciativas de colaboración entre sí como expresión de solidaridad. Dichas relaciones se engloban bajo la denominación "cooperación sur-sur". Esta ha evolucionado a lo largo del tiempo, sujeta a factores como los procesos de integración, los mecanismos de financiamiento, y la inserción de los países del sur en la esfera internacional, entre otros (López, 2014). Una de las ventajas esperables de la cooperación sur-sur es la mayor coincidencia — respecto de la cooperación norte-sur - en los temas de interés, así como en las posibles soluciones a implementar. La cooperación internacional se torna entonces instrumento para aprender de la experiencia de otros países, a partir del cual la globalización facilita la mitigación de sus consecuencias no deseadas (Levi-Faur y Vigoda-Gadot, 2004).

En efecto, la reproducción de políticas públicas o transferencia de políticas es una faceta de la cooperación cada vez más común entre los países en desarrollo. Como señalan Faret et al. (2014: 15): "la cooperación para el desarrollo (...) ha comenzado a acercarse rápidamente a las políticas públicas y sus procesos de construcción”. Jing et al. (2020) afirman que, de hecho, unas de las peculiaridades de la cooperación sur-sur es que en muchos casos se trata de un apoyo no monetario, el cual se traduce, sin embargo, en asistencia técnica o en compartir conocimiento que sea útil para el desarrollo.

La Seguridad Alimentaria y Nutricional (san) es abordada por la cooperación sur-sur, dada su importancia para los países en desarrollo. Conforme a la definición generada en la Cumbre sobre la Alimentación de 1996, entendemos por san el acceso y la disponibilidad por parte de la población de alimentos suficientes, inocuos y nutritivos. Para enfrentar los problemas de inseguridad alimentaria son esenciales políticas que incidan sobre el funcionamiento de los sistemas alimentarios (faO, 2016).

En América Latina, Brasil lleva desde los años 70 desarrollando políticas en pro de la san en su población. Asimismo, forma parte de los países emergentes que han incrementado en mayor medida su presencia en la cooperación sur-sur (Zoccal Gomes y Esteves, 2018). En los años 2000 se producen varios hitos de gran relevancia en Brasil en materia de política en SAN: la creación del Consejo y posteriormente del Sistema Nacional de Seguridad Alimentaria y Nutricional; la puesta en marcha de la estrategia "Hambre Cero", y la aprobación de la Ley Orgánica de Seguridad Alimentaria y Nutricional. El sector público queda entonces como principal garante del Derecho a la Alimentación, el cual se encuentra recogido en la Constitución Federal. Una de las iniciativas concretas desarrolladas en este 
contexto es la creación en 2003 del Programa de Adquisición de Alimentos (PAA). Su objetivo, aprovechar el poder comprador del Estado para el fomento de la Agricultura Familiar (AF), a la vez que se ofrecen alimentos frescos y nutritivos a las poblaciones más vulnerables en establecimientos públicos (MDs, 2014). El PAA brasileño ha recibido mucha atención en la investigación, sobre todo mediante la evaluación de su impacto. En general, se reconoce como una política que ha sido clave para el fortalecimiento de la agricultura familiar en Chile y de la seguridad alimentaria (Berchin et al., 2019). En lo primero, se valoran los efectos en los ingresos y en la diversificación productiva (Petrini et al., 2016; Valencia et al., 2019). Respecto de la san se observa una mejora en la cantidad y variedad de frutas, verduras, legumbres, cereales y lácteos (De Amorim et al., 2016; Teo, 2018).

A la vista de estos antecedentes, otros países de América Latina han explorado las compras públicas a la $\mathrm{AF}$. Chile comienza a desarrollar su propio programa al respecto durante el segundo gobierno de la Presidenta Michelle Bachelet (2014-2018). Aunque el ámbito que se propone es más amplio, en una primera instancia el programa se ha focalizado en la alimentación escolar. Se basa en la colaboración entre el Instituto de Desarrollo Agropecuario (INDAP) y la Junta Nacional de Auxilio Escolar y Becas (junaeb). El primero interesado en facilitar la comercialización de las producciones de los pequeños agricultores, y el segundo en la mejora de la dieta de los estudiantes.

El presente artículo está dividido en tres secciones: en la primera, se describe el caso de la política de compras públicas a la AF de Brasil, con especial enfoque en el Programa de Adquisición de Alimentos; en la segunda, se expone el contexto, origen y contenidos del programa de compras públicas de Chile, y en la tercera se analiza la influencia del programa brasileño en su par chileno a partir de una revisión de los mecanismos de intercambio y cooperación entre Chile y Brasil, sobre todo en materia alimentaria. La información para la elaboración del presente artículo procede de una revisión documental, así como de la recopilación de información primaria mediante entrevistas a informantes clave involucrados tanto el programa brasileño como en el chileno.

\section{El Programa de Adquisición de Alimentos de Brasil}

El paA de Brasil fue establecido por la Ley o 10.696 del 2 de julio de 2003, como parte de la estrategia Hambre Cero. Sus objetivos eran los siguientes: a) asegurar el acceso a los alimentos en cantidad, calidad y regularidad necesaria para atender a las poblaciones en situación de inseguridad alimentaria 
y nutricional; b) contribuir a la formación de centros de acopio estratégicos; c) permitir a los agricultores y agricultoras familiares que almacenen sus productos para que sean comercializados a precios más justos; d) fomentar la inclusión social en las zonas rurales a través del fortalecimiento de la AF, y e) suministrar el mercado institucional de alimentos, incluida la alimentación escolar (CONAB, 2016).

El programa funciona a partir de la compra de los alimentos por el gobierno directamente de los agricultores familiares, asentados de la reforma agraria, comunidades indígenas y demás pueblos y comunidades tradicionales, para la formación de acopios estratégicos y distribución posterior a la población en mayor vulnerabilidad social. Los alimentos tienen como destino las entidades de la red socio-asistencial (guarderías, escuelas, hospitales, Fuerzas Armadas, casa de mayores, etc.), los restaurantes populares, bancos de alimentos y cocinas comunitarias, así como las canastas distribuidas por el Gobierno Federal.Las modalidades de funcionamiento del programa son cinco y se resumen en el Cuadro 1.

\section{Cuadro 1}

\begin{tabular}{|l|l|l|}
\hline MODALIDAD & OBJETIVO & PROVEEDORES \\
\hline $\begin{array}{l}\text { Compra para } \\
\text { donación } \\
\text { simultánea }\end{array}$ & $\begin{array}{l}\text { Atender las demandas locales de complemen- } \\
\text { tación alimentaria, promoviendo el derecho a } \\
\text { una alimentación adecuada. }\end{array}$ & $\begin{array}{l}\text { Individuos, } \\
\text { cooperativas o } \\
\text { asociaciones. }\end{array}$ \\
\hline $\begin{array}{l}\text { Formación de } \\
\text { acopios }\end{array}$ & $\begin{array}{l}\text { Apoyar financieramente la constitución de un } \\
\text { stock de alimentos, lo que facilite la agrega- } \\
\text { ción de valor y la sustentación de precios. }\end{array}$ & $\begin{array}{l}\text { Cooperativas o } \\
\text { asociaciones. }\end{array}$ \\
\hline $\begin{array}{l}\text { Compra } \\
\text { directa }\end{array}$ & $\begin{array}{l}\text { Apoyar la sustentación de precios de un gru- } \\
\text { po de productos, generar stocks públicos de } \\
\text { esos productos y atender el acceso a alimentos. }\end{array}$ & $\begin{array}{l}\text { Individuos, } \\
\text { cooperativas o } \\
\text { asociaciones. }\end{array}$ \\
\hline $\begin{array}{l}\text { Incentivo a la } \\
\text { producción } \\
\text { y consumo } \\
\text { lechero }\end{array}$ & $\begin{array}{l}\text { Asegurar la distribución gratuita de leche a } \\
\text { ciudadanos en vulnerabilidad social y/o inse- } \\
\text { guridad alimentaria y nutricional en los esta- } \\
\text { dos del Nordeste y Minas Gerais. }\end{array}$ & $\begin{array}{l}\text { Individuos, } \\
\text { cooperativas o } \\
\text { asociaciones }\end{array}$ \\
\hline $\begin{array}{l}\text { Compra } \\
\text { institucional }\end{array}$ & $\begin{array}{l}\text { Atención de demandas regulares de consumo } \\
\text { de alimentos por la Unión, Estados, Distrito } \\
\text { Federal y Municipios, los cuales compren con } \\
\text { sus propios recursos financieros. }\end{array}$ & $\begin{array}{l}\text { Individuos, } \\
\text { cooperativas o } \\
\text { asociaciones }\end{array}$ \\
\hline
\end{tabular}

(Fuente: Elaborado a partir de información en MDs, 2014) 
El límite anual de pagos a los agricultores familiares que ofertan al PAA de forma individual para la modalidad de compra con donación simultánea es de R \$ 6.500. Sin embargo, ellos pueden participar a la vez de otros mecanismos similares, como es el Programa Nacional de Alimentación Escolar (PNAE), al cual nos referiremos más adelante en la presente sección. En las otras modalidades del PAA con ofertas individuales, el techo es de R \$ $8.000 \mathrm{y}$ hay límites mayores si se oferta a través de las cooperativas o asociaciones. El PAA es ejecutado con recursos de la Secretaría Especial de Agricultura Familiar y del Desarrollo Agrario (SEAD) y del Ministerio de Desarrollo Social y Agrario (MDSA), en asociación con estados, municipios y con la Compañía Nacional de Abastecimiento (CONAB). La gestión del programa está en manos del mDsa, el Ministerio de Agricultura, Ganadería y Abastecimiento (MAPA), CONAB, el Ministerio de $\mathrm{Ha}$ cienda, el Ministerio de Planificación, Presupuesto y Gestión (MPOG) y el Ministerio de Educación (MEC). El sistema informático SISPAA (Sistema del PAA) acompaña el funcionamiento logístico del programa mediante el almacenamiento de los documentos fiscales correspondientes. Por su parte, el Banco de Brasil es la institución financiera que facilita cuenta vista para los agricultores en el PAA, donde se les depositan los pagos por los productos que venden al sistema.

Brasil fortalece su política de compras públicas a partir de la entrada en vigor de la Ley no 11.947 de 2009 relativa al PNAE. En su artículo 14 se establece que el $30 \%$ de los recursos que el Fondo Nacional de Desarrollo de la Educación (FNDE) dedica al PNAE debe ser utilizado para la adquisición directa de alimentos a la AF, con prioridad en los asentamientos de la reforma agraria, las comunidades indígenas y las afrodescendientes.

Tanto el PAA como el PNAE tienen cobertura en todo Brasil. Sin embargo, se enfocan especialmente en aquellos territorios donde oferta (AF) y demanda (población en situación vulnerable) confluyen. Un ejemplo es el Agreste Meridional del Estado de Pernambuco, el cual fue visitado en 2018 con el fin de levantar información primaria para la presente investigación y por ello se expone en adelante como caso de ejemplo específico para ilustrar de manera más concreta el funcionamiento del PAA.

El paA se inicia en Pernambuco en 2008, abarcando los 11 municipios más pobres del Estado, los cuales pertenecían precisamente al Agreste Meridional. Antes de empezar con las compras se realiza un catastro de las familias que podrían ser proveedoras. A pesar de que los precios eran atractivos, en la primera temporada de ejecución del paA los pagos tardaban meses en realizarse, lo que desincentivaba la participación. En el segundo año de funcionamiento se logró que los pagos fueran a 30 días. En los años siguientes la cobertura se va ampliando, sobre todo a partir de 2012 y de 2015-2016, 
cuando se trabaja ya con 115 municipios. En 2018 la participación es de 108 municipios en todo Pernambuco, pero solamente 90 están ejecutando realmente el paA. Esto es debido a cambios en las bases del programa: por una parte los precios ofrecidos por los productos son menores y, por otra, las exigencias en inocuidad se intensificaron. El último punto ha imposibilitado la participación en el PAA de muchos agricultores que proveían productos elaborados, carnes y/o derivados animales. Varios municipios del Agreste Meridional son eminentemente lecheros, por lo que muchos de sus productores se vieron "expulsados" del paA. En todo caso, el problema no es solamente si los productores tienen la capacidad de cumplir (o no) con los nuevos requisitos de inocuidad, sino que en la región del Agreste, solo tres municipios cuentan con capacidad fiscalizadora al respecto, la cual es imprescindible. En relación a los precios de compra del paA, hasta 2016 estos se encontraban por encima de los del mercado, sin embargo se ajustaron a la baja mediante una tabla estandarizada por productos.

El Instituto Agronómico de Pernambuco (IPA), junto con las municipalidades, se encarga de gestionar la operativa del PAA en el Agreste pernambucano. El corazón del proceso es la compra de los alimentos. Para ello los agricultores acuden a un punto de acopio acordado, donde profesionales del IPA van registrando en planillas lo que cada productor entrega. Dicha información se traslada a la sede central del IPA, para luego derivarse al Gobierno Federal a través de un soporte computacional y también en papel. Una vez aprobada la validez de la información, se emite el pago mensual a cada agricultor con cargo a su cuenta del Banco de Brasil. Los productos entregados en el punto de acopio son trasladados de inmediato a los centros públicos asociados al PAA, donde son recibidos por un profesional del mismo designado para tales fines, quien los revisa. Para que la oferta y la demanda vinculada al paA concuerden, las compras están precedidas por un proceso de planificación anual. Así, cada agricultor tiene claridad sobre cuáles son los productos (y las cantidades) que debe entregar. Por otro lado, extensionistas del IPA realizan acompañamiento técnico a los agricultores en el PAA.

Pese al punto de inflexión en el que se encuentra el paA y que se agravó en 2019 con el cambio de gobierno en favor del Presidente Jair Bolsonaro, en opinión de agricultores y profesionales de apoyo, el programa ha tenido un impacto muy positivo en la AF del Agreste pernambucano."El programa ayuda mucho. Estamos agradecidos. No solo por los agricultores, sino por las familias. Ayudó al pueblo. Mi vida mejoró en un 100 por 100. Antes no teníamos a quién vender" (O. Araújo de Lima, agricultor, comunicación personal, marzo 2018). Eso ha llevado a la fidelización de los productores participantes, independiente de su género. 
El PAA "es verdaderamente incluyente para las mujeres [agricultoras], incluso con los precios más bajos, [ellas] siguen ofertando [sus producciones] porque es una compra segura y garantía de renta” (I. Mesquita, extensionista, comunicación personal, marzo 2018).

Por otro lado, el menú en las escuelas y otros centros públicos asociados en el paA se vio muy enriquecido. Antes del programa primaban los alimentos procesados, como las pastas o las galletas. En la actualidad las preparaciones se basan en productos frescos de procedencia local. El hecho de que los productos que consumen los niños y niñas de la comunidad provengan de sus predios es algo que fue destacado por los agricultores participantes en el PAA entrevistados como una fuente de orgullo y un incentivo. Por otro lado, se señalaba que se genera un sentido de identidad territorial también en los estudiantes.

\section{Las Compras públicas a la AF eN Chile}

\subsection{Panorama general de la AF y la alimentación en Chile}

Chile es un país con características geográficas peculiares. Tiene 4.200 kms. de largo, pero con un ancho máximo de 375 kms.; cercado en el Este por cordilleras - de Los Andes y de la Costa-; al Norte su frontera es el Desierto de Atacama; al Sur la Patagonia chilena, y al Oeste el Océano Pacífico.Esta morfología proporciona una amplia variedad climática a lo largo del territorio, que va desde el tipo desértico en el Norte, con precipitaciones promedio de menos de $1 \mathrm{~mm} / \mathrm{año}$, al templado lluvioso frío en el extremo sur, con lugares cuyas lluvias superan los $5.000 \mathrm{~mm} / \mathrm{año} \mathrm{(ODEPA,} \mathrm{2016).} \mathrm{La}$ actividad agrícola se concentra en la zona central y centro-sur del país (de la Región de Valparaíso a la del Bío-Bío), la cual goza de clima mediterráneo con lluvia invernal.

Las explotaciones agrícolas chilenas pueden ser clasificadas casi en su totalidad como micro y pequeñas empresas (Aedo y Alvear, 2010). Sin embargo, las pocas empresas medianas y grandes concentran la mayor parte de las hectáreas productivas. El tamaño de las explotaciones agrícolas está muy relacionado con su manera de abordar la comercialización. En este sentido, Ríos y Torres (2014) afirman que la agricultura chilena se caracteriza por una marcada dualidad entre una minoría de empresas medianas y grandes exportadoras, y una mayoría de micro y pequeños productores orientados al mercado local. Incluso dentro de la primera categoría existen contrastes. InDAP divide a los pequeños agricultores chilenos en "multiactivos", que 
necesitan complementar ingresos con actividades distintas a la explotación agrícola, en oposición a los "comerciales", focalizados en su producción. El primer grupo concentra el $61 \%$ de los pequeños productores y el segundo el $39 \%$ restante (Rimisp y Demoscópica, 2017). Echeverría et al. (2012) demostraron que en Chile los agricultores orientados al mercado nacional tienen un notorio atraso en uso de tecnologías, acceso a financiamiento e incluso a programas públicos respecto de los exportadores.

De forma específica, en Chile se estima hay 262.000 explotaciones campesinas, que aportan $61 \%$ del empleo agrícola, utilizan $44 \%$ de la superficie útil y representan un $22 \%$ del valor de la producción (INDAP, 2017). Respecto de los rubros, su presencia es especialmente relevante en la horticultura, floricultura, cultivos anuales, producción caprina, apícola y bovina. La fruticultura es el rubro que más ha aumentado en superficie y que mayor contribución al PIв realiza, así como al comercio exterior silvoagropecuario chileno supone (ODEPA, 2016), en él la participación de la pequeña agricultura es secundaria (alrededor del 20-25\% de la superficie). Sin embargo, conforme a Sáez et al. (2015) hay que exceptuar las denominadas como "frutas de huerta", las cuales debido a las limitaciones de su post cosecha son poco exportadas y se encuentran principalmente en manos de la $\mathrm{AF}$ (ejemplo, melones y sandías).
A pesar de su importancia para proveer los alimentos internamente, la $\mathrm{AF}$ chilena todavía tiene problemas severos como la alta informalidad, dificultades en el acceso a sistemas de riego, la discontinuidad de la oferta, bajo uso de tecnologías de información y productivas, entre otros, lo que a su vez complica el acceso y competitividad en los mercados (Boza et al., 2015, 2016, 2019). Respecto de lo último, los productores aluden a la falta de canales para la venta como una restricción adicional (ODEPA, 2016).

Las limitaciones de la distribución de alimentos frescos en el mercado nacional no suponen un problema únicamente para los productores, sino que además restringen las posibilidades de abastecimiento de la demanda. Lo anterior efectivamente condiciona el comportamiento alimentario de los consumidores chilenos (Espacio y Fomento, 2016); es decir, es un factor que determina su entorno o ambiente alimentario (Cerda et al., 2016). Solo el 5\% de la población chilena tiene una dieta que pudiera ser considerada como saludable (Universidad de Chile, 2014). En el caso específico de los escolares, entre un 45 y un $50 \%$ padece sobrepeso u obesidad (Junaeb, 2018). El nivel socioeconómico condiciona tanto en la infancia como en la edad adulta la prevalencia de sobrepeso (Azar, 2015; Minsal, 2017). No obstante, en el caso de los estudiantes, los hábitos alimentarios inadecuados son transversales (Castillo et al., 2016). 
Por tanto, nos encontramos por un lado con un sector atomizado, con alta participación de pequeños agricultores. Estos presentan, entre otras, limitaciones para comercializar sus productos. Asimismo, la demanda se caracteriza por su inclinación a alimentos no saludables, lo que deriva en una alta incidencia de sobrepeso y obesidad. Políticas que promuevan mayor vinculación entre la oferta de productos frescos de la AF y la demanda constituyen una alternativa que lograría al menos mitigar ambas situaciones.

\subsection{Estrategia pública para el sistema alimentario chileno}

Tradicionalmente, el abordaje del sector público chileno hacia los agricultores se ha basado en mejorar y aumentar su producción, con el fin de incrementar sus ingresos. Asistencia técnica, crédito y capacitaciones han sido los instrumentos clave (Sotomayor et al., 2011). Sin embargo, en el segundo gobierno de la Presidenta Michelle Bachelet se acrecentó la preocupación por la comercialización de los productos de la AF. Así fue declarado por la propia Mandataria en la celebración del "Día del Campesino", en julio de 2014, en el Palacio de la Moneda. En la oportunidad, afirmó que "se ha iniciado el trabajo en tres líneas prioritarias para los pequeños productores agrícolas: enfrentar la sequía, mejorar los canales de comercialización y fomentar la organización campesina”.
A nivel práctico, este cambio de enfoque se vio reflejado principalmente en las acciones de InDAP. En sus lineamientos estratégicos 2014-2018, se explicita la mayor inserción de la AF en el mercado interno como un objetivo clave. Se declara que se ampliarán las capacidades institucionales para poder abordar la materia. El Programa de Comercialización de Indap es la expresión de dichas intenciones. Entre las iniciativas desarrolladas bajo su alero destacan: sello "Manos Campesinas”, Expo Mundo Rural y otras ferias, tiendas "Mundo Rural”, tienda virtual y compras públicas.

En el caso del sello "Manos Campesinas", los productos que ostentan dicha distinción deben, entre otros, haber sido obtenidos por pequeños productores de forma artesanal. Conforme a las normas técnicas del sello, esto último se relaciona con: i) la participación activa del productor a lo largo del proceso, ii) que una parte significativa del proceso productivo sea realizada a mano, y iii) que la mayoría de las materias primas sean de producción propia. El programa comenzó en 2015, con una experiencia piloto que incluía algunos productos y zonas del país. En la actualidad su cobertura es nacional y considera productos primarios vegetales, animales, elaborados e incluso artesanías. Respecto de las tiendas "Mundo Rural", la primera fue abierta a mediados de 2016, en el centro de Santiago, con una oferta de más de 400 productos. Todas las tiendas que operan dentro de 
la red utilizan una marca registrada común, así como una imagen corporativa. Los proveedores deben cumplir con los requisitos en la Ley Orgánica de INDAP, además de las especificaciones técnicas y legales del producto a ofertar. Por su parte, la promoción se materializó durante el período 20142018, esencialmente en la Expo Mundo Rural —que reunía por varios días a pequeños agricultores y artesanos, exponiendo y vendiendo sus productos, así como se realizaban actividades temáticas-y otras ferias abiertas al público, como el Festival Ñam.

En el caso específico de las compras públicas a la $\mathrm{AF}$, está bajo el alero del Programa de Comercialización de INDAP, pero a su vez - aunque no exclusivamente - se ha vinculado al Plan Contrapeso coordinado por Junaeb. Dicho Plan se lanzó a fines de 2016, teniendo por objetivo reducir la alta obesidad infantil presente en el país. Se diseñó mediante el trabajo colaborativo de distintas instituciones dedicadas a aspectos relativos a la salud, la nutrición, el deporte, la educación y la infancia. Contrapeso se concreta a través de 50 medidas en cinco ejes fundamentales: alimentación, actividad física, innovación y tecnología, educación alimentaria y monitoreo. Dentro de sus medidas - además de las compras públicas a la $\mathrm{AF}$-, algunas de las más destacables son: i) la progresiva disposición de buffet de ensaladas (o salad bar) para los estudiantes en el Programa de Alimentación Escolar (PAE) pertenecientes a la educación media; ii) el cambio de la sal procesada por sal de mar en los menús de los establecimientos educacionales y la restricción de los azúcares en la dieta; iii) la disposición de pantallas en los casinos donde se muestre el menú del día; iv) inclusión de mayor actividad física en los horarios de recreo; v) entrega a los estudiantes de una pulsera inteligente que informe sobre la actividad física realizada e incentive a llevar a cabo más, y vi) sustitución de productos que contengan sellos de advertencia nutricionales. La mayoría de estas medidas no han sido posibles de implementar sobre todo por limitantes logísticas, pero se trabajó por una intensificación en la educación alimentaria.

\subsection{El programa de compras públicas a la AF en Chile}

El programa de compras públicas de INDAP tiene por objeto facilitar que la pequeña agricultura chilena tenga la capacidad de insertarse en los canales institucionales de compras. Hasta el momento, el programa se ha focalizado en la alimentación escolar a través de la colaboración con Junaeb, aunque hay otros mercados respecto de los que se ha trabajado. Por ejemplo, la inserción de productores en el catálogo de proveedores preferente del sector público - Convenio Marcoen la categoría de alimentación.

Junaeb es la institución en Chile que presenta una mayor demanda de servicios alimentarios, no solo a través 
del PaE, sino también para los Programas de Alimentación de Párvulos (PAP) — con el apoyo de la Junta Nacional de Jardines Infantiles (JunjI) y la Fundación INTEgra - y para las becas en educación superior (Sáez et al., 2015). La Ley o 19.886, que regula en Chile los contratos administrativos de suministros y las prestaciones de servicios, establece como una de las modalidades principales para las compras institucionales la celebración de licitación pública. En el caso del paE de Junaeb, ese es el mecanismo que se utiliza para concretar el aprovisionamiento. El territorio nacional se segmenta en tres partes; para cada una de ellas se realiza licitación cada tres años. Los postulantes deben presentar el precio por ración, los costos de implementación y garantía de seriedad de la oferta. Las concesionarias adjudicadas son responsables de la disponibilidad de los alimentos y su distribución a los centros educativos. Las condiciones que tengan estos para cocinar y almacenar alimentos, determinan las características del servicio (u oferta) que les provee la concesionaria (esencialmente el nivel de preparación de los ingredientes).

Junaeb, en tanto, se encarga de definir las bases operativas generales y la supervisión del sistema. Por ello, cualquier cambio en los requisitos de las licitaciones debía pasar por su respaldo. En este sentido, dada la magnitud del PAE, para tener mayores antecedentes se decidió, en primer lugar, llevar a cabo algunas experiencias piloto de compras públicas a la AF en ciertas regiones y para algunos productos: Coquimbo (tomates), O'Higgins (limones, naranjas y zapallo de guarda), Maule (frutas y hortalizas), Araucanía (frutas) y Los Lagos (frutas y hortalizas). En ningún caso las concesionarias desaparecían, sino que los productores dentro del piloto les proveían a las mismas en parte de su demanda. Por tanto, los proyectos piloto fueron ejecutados para preparar la entrada en vigencia de las compras a la producción local, y en concreto a la AF (INDAP-Junaeb, 2017), incluyendo conocer en profundidad los modelos de abastecimiento de las concesionarias, las necesidades de los pequeños agricultores para poderse insertar en el sistema e identificar potenciales interesados.

En 2017 se publica la primera licitación donde se exige a las concesionarias una cuota de compra a pequeños productores locales ${ }^{1}$. En general, $5,25 \%$ del total facturado, aproximadamente $15 \%$ del gasto en insumos o 3,5\% si la compra se realiza en zonas rezagadas ${ }^{2}$, aproximadamente $10 \%$ del gasto en insumos. El porcentaje es intermedio

1 Se entiende por ello a los que realicen sus actividades en la misma Región en la que se van a entregar las raciones a los escolares. Por otro lado, en los productos primarios, es InDAP quien avala la condición de pequeño productor, o en caso de no ser posible SERCOTEC u otra institución.

2 Junaeb establece que como "zona rezagada" se entenderá aquellas indicadas en los Decretos no 1116 y 1459 de 2014 y no 1929 de 2015, del Ministerio del Interior y Seguridad Pública de Chile. 
cuando se trata de una compra mixta (zonas rezagadas y no rezagadas). Más allá de la definición de los porcentajes de compra, del concepto de zonas rezagadas y de qué proveedores son aptos para ser considerados como pequeños productores locales, Junaeb no incide mayormente en las condiciones de compra. La implementación de las nuevas licitaciones se planteó de forma gradual, como se muestra en el Cuadro 2.

Cuadro 2

\begin{tabular}{|l|l|l|}
\hline AÑo & REGIONES & ENTRADA EN VIGENCIA \\
\hline 2017 & $\begin{array}{l}\text { Atacama, 1/3 RM, O’Higgins, } \\
\text { Biobío y Aysén. }\end{array}$ & $\begin{array}{l}\text { Agosto 2017: O’Higgins y } \\
\text { Biobío. } \\
\text { Marzo 2018: Atacama, Aysén y } \\
1 / 3 \text { RM. }\end{array}$ \\
\hline 2018 & $\begin{array}{l}\text { Coquimbo, Valparaíso, 1/3 } \\
\text { RM, Araucanía y Magallanes. }\end{array}$ & $\begin{array}{l}\text { Agosto 2018: Coquimbo, Valpa- } \\
\text { raíso, 1/3 RM y Araucanía. } \\
\text { Marzo 2019: Magallanes. }\end{array}$ \\
\hline 2019 & $\begin{array}{l}\text { Arica-Parinacota, Tarapacá, } \\
\text { Antofagasta, 1/3 RM, Maule, } \\
\text { Los Ríos y Los Lagos. }\end{array}$ & $\begin{array}{l}\text { Marzo 2019: Arica-Parinacota, } \\
\text { Tarapacá, Antofagasta, } 1 / 3 \text { RM, } \\
\text { Maule, Los Ríos y Los Lagos }\end{array}$ \\
\hline
\end{tabular}

El programa de compras públicas de INDAP define cinco categorías de productos prioritarios: hortalizas y papas, frutas frescas, legumbres, quínoa y miel. Sin embargo, en las licitaciones para la alimentación escolar se ha podido avanzar en hortalizas y frutas. En el caso de legumbres y granos, resulta difícil que los pequeños agricultores puedan competir con el precio que a las concesionarias les ofrecen otros proveedores que, en gran medida, importan. En el caso de la miel no se ha logrado visualizar cómo insertarla de forma eficiente en el menú escolar. Por otro lado, para cumplir con el porcentaje de compra local requerido, las bases de las licitaciones no excluyen de la contabilidad otros productos distintos a los de la AF, como pescado, productos elaborados o incluso pan. En general, el modelo de compras de las concesionarias les ha dificultado, en cierta medida, insertar las compras a la $\mathrm{AF}$, dado que en la mayor parte de los casos estas se encuentran centralizadas. Las decisiones se toman para grandes volúmenes, con lo que se aprovechan las economías de escala. De hecho, dado que Junaeb no ha incrementado especialmente el precio que paga por ración, las concesionarias no pueden permitirse aumentar 
sus costos si pretenden mantener su margen habitual de ganancia.

El potencial de compras locales de Junaeb está estimado en 28 mil millones de pesos chilenos. Sin embargo, en sus dos primeros años de funcionamiento se calcula que las compras a la agricultura familiar han alcanzado solamente mil millones de pesos. Esta diferencia se debe a que las concesionarias compensan la cuota de compras locales, en gran medida, a través de productos elaborados, como pan. Para tratar de mitigar esta situación, Junaeb está revisando sus minutas, incorporándoles mayor identidad local.

\section{DisCuSión SOBRE LA INFLUENCIA BRASILEÑa EN LAS COMPRAS PÚBLICAS A LA AF CHILENA}

En las relaciones internacionales, es común el debate acerca del término soft power definido por Joseph Nye (1990), de forma amplia, como la capacidad de un país para persuadir sin la fuerza o la coacción. Es decir, un tipo de poder que no implicaría el uso de la fuerza física y sí la capacidad de convencimiento. Cuando se habla de influencia de un país sobre otro se está presuponiendo también persuasión, o sea, un tipo de soft power. La forma como Brasil puede haber influido en el establecimiento de la política de compras públicas a la $\mathrm{AF}$ en Chile parece, a priori, ajustarse a un ejemplo de soft power basado en el predominio de una historia, en este caso de una política pública exitosa, de un país que comparte características en común con Chile. Este proceso podría encajar también en un modelo de cooperación Sur-Sur, protagonizado, en general, por países que comparten contexto político, hábitos, similares problemas, y - ¿por qué no?- soluciones para aquellos.
En este sentido, Reich y Lebow (2014: 6) apuntan que "la influencia efectiva se basa en la persuasión (...) La persuasión depende de los valores compartidos y las prácticas aceptables, y cuando funciona, ayuda a construir identidades comunes que pueden hacer que la cooperación y la persuasión sean más probables en el futuro. La influencia de este tipo también se beneficia de las capacidades materiales, pero no es una función de ellos. Está restringida a objetivos comunes y requiere habilidades políticas considerables. Depende de líderes y diplomáticos sofisticados, discursos compartidos con los Estados destinatarios, defensa de políticas que se basan en precedentes y voluntad de permitir que otros ayuden a dar forma y poner en práctica iniciativas".

Por tanto, la acción de influenciar es vía persuasión, convencimiento, o sea, principalmente a través del diálogo. Segundo, hay una condición previa al mecanismo de persuadir que es la base de valores comunes (que 
se pueden fundamentar en el pasado común - ej. todos los países suramericanos fueron colonias - contextos económicos semejantes, coyunturas políticas, etc.), además de en prácticas aceptables y admisibles (ciertamente no es aceptable la tortura y privación de derechos, entre otras). Así que a partir de esa condición, de esa base común de valores y de prácticas plausibles, la probabilidad de generar arreglos cooperativos duraderos y estables en el tiempo es más grande. Otro aspecto son las capacidades materiales, es decir, el nivel de recursos disponibles. Si bien es relevante, la influencia no se muestra a partir de ellos. Aparte está la necesidad de compartir discursos con los países "receptores", ayudando a que la influencia tome forma y se concrete en una política pública, por ejemplo.

Además de compartir intereses generales, es relevante que los países tengan situaciones semejantes para facilitar el intercambio de ideas. Brasil ha logrado erradicar la extrema pobreza (hasta inicios de los años 2000) y el hambre crónicos. En ese proceso se ha conseguido aumentar la participación de la AF en la economía, lo que fue esencial para la disminución de las desigualdades entre las regiones del país y proporcionó alimentos más frescos para los niños en las escuelas. Chile ha logrado también erradicar el hambre, que era un problema común en AL, sin embargo, hoy se encuentra en la condición opuesta, la de malnutrición por exceso. Por otro lado, su
AF se enfrenta a importantes limitaciones, como es el acceso a mercados y su propia viabilidad.

Respecto de los canales específicos de intercambio de información, existen encuentros, comitivas, fórums, redes académicas y otras instancias en que Chile ha tenido la posibilidad de entrar en contacto con la gestión, logística y funcionamiento de programas brasileños que fomentan la participación de la agricultura familiar en la economía. Además, por supuesto, se debe considerar la coordinación de los países en grupos de cooperación e integración (MERCosur, Aladi, etc.), así como la participación en organizaciones internacionales, donde el intercambio se hace parte del día a día.

Un hito clave en este sentido es la constitución de la Reunión Especializada de Agricultura Familiar del Mercosur (REAF) en 2004. La REAF es un fórum donde se encuentran productores familiares, organizaciones e instituciones rurales de la región, para compartir experiencias e intercambiar conocimientos. En el momento de su creación, el gobierno del Presidente Lula da Silva completaba un año y ya había puesto en marcha las compras públicas a la AF en Brasil. De hecho, en el xIv Encuentro REAF, en noviembre de 2010, se estableció el programa regional "Intercambio de experiencias sobre modelos de gestión de las políticas de compras públicas de la agricultura familiar", financiado por la Agencia Brasileña de Cooperación (ABC). 
Este programa fue dividido en seminarios y visitas de comisiones a países que tenían experiencias que aportar. El primer módulo fue en los estados de Goiás, Distrito Federal y Pernambuco, Brasil, en agosto de 2011; el segundo en Mendoza y San Juan, Argentina, en abril de 2012; el tercero en Montevideo, Uruguay, en junio de 2013, y el cuarto se realizó en Brasilia, Brasil, en noviembre de 2013. Chile recibió la segunda versión del programa en diciembre de 2014; después Quito, Ecuador, en 2015, y finalmente Venezuela, también en 2015. En 2016, el programa fue finalizado con el "Taller REAF MERCosur: Balance del i y ir Programa de Intercambio Regional de las Compras Públicas de la Agricultura Familiar" en Asunción, Paraguay.

Sobre las actividades de la comitiva de REAF MERcosur, en el estado de Pernambuco en 2011, la agenda incluía visitas a entidades beneficiarias del programa, al centro de acopio y distribución de los alimentos, al consejo de los agricultores, a una unidad productiva y a productores de forma particular. El objetivo de la visita era "auxiliar con informaciones a los miembros de la comitiva del Mercosur que visitan las acciones del Programa de Adquisición de Alimentos, en Pernambuco, sustentadas en su operativa por el IPA, órgano vinculado a la Secretaría de Agricultura, responsable por la prestación de servicios de investigación, asistencia técnica y extensión, prioritariamente a los agricultores familiares del Estado (...) visitar entidades socio asistenciales que preparan los alimentos donados para la oferta de comidas a personas en situación de inseguridad alimentaria y nutricional, en los municipios más pobres".

Durante la visita, el viceministro de Agricultura de Paraguay, Andrés Werhle, destacó que "la experiencia brasileña ciertamente es una referencia para ser evaluada” y subrayó que "en toda América Latina, a pesar de las diferencias, las cuestiones como la soberanía alimentaria, la comercialización y los intermediarios están presentes" (IPA, 2011). En el mismo tono, de acuerdo con Juan Guido Vidal y Juan Jiménez ${ }^{4}$, representantes de Chile en REAF, Brasil llevó el tema de las compras públicas de alimentos a la AF a la agenda regional y Chile lo toma y lo trae internamente.

Otro canal para la influencia brasileña sobre la política de compras públicas a la AF en Chile son los estudios ejecutados por los expertos nacionales. En este sentido destaca el trabajo de Luis Sáez, profesor del Departamento de Gestión Agraria de la Facultad Tecnológica de la Universidad de Santiago de Chile (USACH), que por demanda del Gobierno en la época y del movimiento conocido como Slow Food, con financiamiento de la Fundación Ford, coordinó la elaboración del documento "Mercados Públicos de Alimentos

3 Información extraída de documentos internos facilitados por personal de Instituto Agronómico de Pernambuco (IPA).

4 Comunicación personal, 27 de abril de 2018. 
en Chile y recomendaciones para la inclusión de la Agricultura Familiar Campesina”, publicado en 2015. En la investigación, fueron hechas entrevistas a responsables, coordinadores y profesionales de organismos internacionales (sobre todo faO) respecto del funcionamiento de los programas de compras públicas en Brasil y la participación de la AF. De hecho, los autores dedican un anexo para describir experiencias internacionales en el tema, incluida la de Brasil, que destacan como uno de los "principales países en la inclusión de la AF en el proceso de compras públicas" (Sáez et al., 2015:142).El profesor Luis Sáez afirma que, en efecto, Brasil es un referente para Chile en compras públicas a la AF. Sin embargo, señala igualmente que la adaptación para el caso chileno no se hizo tomando todos los elementos brasileños.

En efecto, existen marcadas diferencias entre cómo se han ejecutado las compras públicas a la $\mathrm{AF}$ en Brasil y cómo se ha llevado a cabo hasta ahora el programa chileno. En primer lugar, la intermediación es una diferencia esencial. En Chile son las concesionarias las encargadas de adquirir parte de sus insumos a la AF, mientras que en Brasil ese tipo de intermediarios no existen. De hecho, son los propios agricultores los que entregan directamente sus productos a los encargados públicos de gestionar el programa en su territorio. Como consecuencia, es esperable que el nivel de conocimiento

5 Comunicación personal, 2 de mayo de 2018. por parte de los agricultores sobre los programas de compras públicas, así como su implicación e identificación con ellos, sea muy dispar entre ambos casos.

Por otro lado, la magnitud de los dos programas es también muy diferente. En lo más evidente, la población de Brasil es 12 veces superior a la de Chile. Las dimensiones del territorio son también muy distintas, Brasil es diez veces más extenso que Chile. Asimismo, Brasil está dividido en unidades federales con alto nivel de autonomía, mientras que la administración territorial chilena es mucho más centralizada. En consecuencia, los volúmenes que potencialmente pueden llegar a movilizar ambos programas son obviamente muy distintos. Además, las compras públicas en Brasil tienen una historia mucho más dilatada que en Chile, donde aún es una política novedosa.

Finalmente, otra diferencia importante a señalar entre los dos casos es la inclusión de la AF como proveedor de las compras públicas en la regulación con carácter vinculante. En Brasil, como ya fue señalado anteriormente, en el reglamento del pNaE se establece que un mínimo del $30 \%$ de las compras de alimentos para las escuelas debe venir de la producción de los agricultores familiares. En Chile no hay hasta el momento ningún tipo de norma que establezca esa obligatoriedad y sí un poder discrecional del gobierno central para cada licitación del paE. Por tanto, la estabilidad del 
programa es menor que en Brasil. No haber establecido aún mecanismos tan estrictos puede deberse, por un lado, a lo incipiente del programa en Chile, así como, por otro, a la incertidumbre acerca de la capacidad de la AF de responder con una oferta adecuada.

\section{Consideraciones finales}

El objetivo de este artículo no ha sido, en ningún caso, evaluar la política de compras públicas en Chile o en Brasil. Tampoco afirmar qué país ha hecho mejor las cosas o generar recomendaciones en ese sentido. El propósito fue observar la influencia que Brasil ha tenido sobre otros países de la región —en este caso Chile- en sus políticas relacionadas con la sAN, considerando en específico las compras públicas a la AF. Todo ello dentro del contexto de la cooperación Sur-Sur, concretada en compartir experiencias y, por tanto, transferir modelos para poder atender problemas públicos.

En este sentido fue necesario apuntar a cómo el programa se desarrolló en los dos países para poder, posteriormente, respaldar el análisis de la influencia brasileña. Primero, Brasil ya cuenta con un programa de compras públicas a la $\mathrm{AF}$ asentado, tanto jurídicamente cuanto en cuestiones de presupuesto, asistencia técnica y logística. En Chile, las compras públicas a la $\mathrm{AF}$ han sido iniciadas muy recientemente. Para algunos analistas, no es de hecho aún una política pública fuertemente establecida y sí más bien una demostración de buena intención del gobierno de Michelle Bachelet.
Otra gran diferencia es que la venta ocurre en Chile a través de concesionarias, empresas que ganan las licitaciones y proveen de raciones a las escuelas. A ellas es a quienes se les exige un porcentaje de compras a la AF. Las dimensiones que por ahora dichas compras han alcanzado en Chile son prácticamente testimoniales comparadas con los volúmenes de Brasil.

En todo caso, pese a las diferencias se observa la influencia de Brasil respecto de Chile. Se adoptó el término influencia porque no se firmó ningún acuerdo o convenio de cooperación bilateral concreto. Sin embargo, existieron vías de contacto entre representantes de ambos países, donde pudieron intercambiar experiencias. Todo esto se vio facilitado por las prioridades y problemáticas comunes.

Por tanto, la influencia brasileña se materializa en dictar la agenda, o más conocido por su término en inglés agenda setting. Lo anterior se refiere a la capacidad de un actor de hacer que un tema tome relevancia para ser discutido por otros. En relaciones internacionales, es muy común la presencia de un agenda setter en los fórums, encuentros, conferencias, etc. En general, es un país suficientemente "fuerte" en 
un determinado tema, que logra impulsarlo en la discusión, colocándolo en primer plano.

De esta manera, a pesar de no haber aún evaluaciones sobre la política de compras públicas en Chile, el tema está presente, fue internalizado, debatido, diseñado y creada una estrategia para materializarlo. Por tanto, Brasil ha cumplido su papel de agenda setter. En el proceso, Chile ha adaptado la idea general a sus propias condiciones.

\section{Bibliografía}

Aedo, M. \& Alvear, J.J. (2010). Micro y Pequeña Empresa Agropecuaria en Chile: Criterios para una Focalización Eficiente de las Políticas para el Sector de acuerdo al VII Censo Agropecuario. Santiago: Indap.

Azar, A.; Franetovic, G.; Martínez, M. y Santos, H. (2015). "Individual, social and environmental determinants of overweight and obesity among Chilean adolescents". Revista Médica de Chile, vol.143, núm. 5 .

Berchin, I.; Nunes, N.; de Amorim, W.; Alves, G.; da Silva, F.; Fornasari, V.; de Andrade Guerra, J. (2019). "The contributions of public policies for strengthening family farming and increasing food security: The case of Brazil". Land Use Policy, vol. 82.

Boza, S.; Cortés, M. y Guzmán, F. (2015). "Caracterización de pequeños empresarios agrícolas beneficiarios de programas de desarrollo local en la Región Metropolitana, Chile". Revista IDESIA, vol. 33, núm.1.

Boza, S.; Cortés, M. y Muñoz, T. (2016). "Estrategias de desarrollo rural con enfoque de género en Chile: el caso del programa Formación y Capacitación para Mujeres Campesinas”. Revista Civilizar, vol.16, núm. 30.

Boza, S.; Mora, M.; Osorio, F. y Muñoz, J. (2019). "Family farmers' reluctance toward incorporating into the formal economy". Agricultural and Resource Economics, vol. 18, núm. 2.

Castillo, V.; Escalona, J. y Rodríguez, C. (2016). "Food habits in Chilean schoolchildren. Comparative analysis by type of educational establishment". Revista Chilena de Nutrición, vol. 43, núm. 11.

Cerda, R.; Egaña, D.; Gálvez, P. y Masferrer, D. (2016). Marco conceptual sobre los factores condicionantes de los ambientes alimentarios en Chile. Santiago: Universidad de Chile y Minsal.

Conab (2016). Programa de Adquisición de Alimentos de la Agricultura Familiar PAA. Recuperado el 16 de enero de 2018 de http://www.conab.gov.br/OlalaCMS/ uploads/arquivos/16_06_17_10_22_53 cartilha_ep.pdf

De Amorim, A.; De Rosso, V. y Bandoni, D. (2016). "Acquisition of family farm foods for school meals: Analysis of public procurements within rural family farming published by the cities of São 
Paulo state". Revista de Nutricao, vol. 29, núm 2.

Espacio y Fomento (2016). Estudio para el desarrollo de los mercados locales para mejorar el acceso a frutas y hortalizas frescas en las regiones Metropolitana y del General Libertador Bernardo O'Higgins. Santiago: ODEPA.

FAO (2016). Mejoramiento de los sistemas alimentarios para la nutrición y la salud. Roma: FAO.

Faret, P. y Stevens, C. (2014). Cooperación Internacional y Políticas Públicas de Seguridad Alimentaria y Nutricional. FAO.

INDAP (2017). INDAP en cifras. Recuperado el 10 de mayo de 2018 de: https://www.indap.gob.cl/ biblioteca/documentos-indap/!k/ indap-en-cifras-(primer-semestre-2017)

INDAP-Junaeb (2017). Programa de compras públicas de INDAP - JUNAEB: Información y cifras relevantes. Recuperado el 6 de marzo de 2018 de https://www. camara.cl/pdf.aspx?prmID=102830\&prmTIPO=DOCUMENTOCOMISION.

IPA (2011). Mercosul e África conhecem experiências de compras públicas do Brasil. Recuperado el 5 de abril de 2018 de http://www.ipa.br/noticias_detalhe. php?idnoticia $=2237 \&$ secao $=1$

Jing, Y.; Mendez, A. y Zheng, Y. (2020). New development assistance. Emerging economies and the new landscape of development assistance. Singapore: Palgrave Macmillan.

Junaeb (2018). Informe Mapa Nutricional. Recuperado el 21 de marzo de 2019 de https://www.junaeb.cl/wp-content/
uploads/2013/03/MapaNutricional_2018. pdf

Levi-Faur, D. y Vigoda-Gadot, E. (2004). International Public Policy and Management. Policy Learning Beyond Regional, Cultural, and Political Boundaries. New York: Marcel Danker.

López, S. (2014). “Cronología e Historia de la Cooperación Sur-Sur: Un aporte desde Iberoamérica”. Programa Iberoamericano para el Fortalecimiento de la Cooperación Sur-Sur, Documento de trabajo no. 5.

MDS (2014). PAA: Manual Operativo - Modalidade Compra com Doação Simultâea. Recuperado el 20 de marzo de 2018 de http://www.mds.gov.br/webarquivos/publicacao/seguranca_alimentar/ manualPAA.pdf

Melillo, C. (s. f.). Inclusión de la agricultura familiar campesina (AFC) como proveedor de mercados públicos de alimentos [PPT]. INDAP.

MINSAL (2017). Encuesta Nacional de Salud ENS Chile 2016-2017: Primeros resultados. Recuperado el 17 de octubre de 2017 de http:/www.minsal.cl/ wp-content/uploads/2017/11/ENS-201617_PRIMEROS-RESULTADOS.pdf Nye, J. (1990).Bound to Lead: The Changing Nature of American Power. New York, US: Basic Books.

ODEPA (2016). Estudio sobre desafíos de políticas sectoriales para una agricultura competitiva y sostenible al año 2030, visión desde las regiones. Santiago: ODEPA.

Petrini, M.A.; Rocha, J.; Brown, J. y Bispo, R. (2016). "Using an analytic hierarchy 
process approach to prioritize public policies addressing family farming in Brazil". Land Use Policy, vol. 51.

Reich, S. y Lebow, R. (2014). Good-bye hegemony! Power and influence in the global system. New Jersey: Princeton University Press.

Rimisp y Demoscópica (2017). Línea base de los usuarios de INDAP 2015. Santiago: INDAP, Rimisp y Demoscópica.

Ríos, S. y Torres, G. (2014), "El sector agropecuario en la región de Los Lagos y el paradigma Chile potencia alimentaria: desafíos para la política agraria nacional". Mundo Agrario, vol. 15, núm. 29.

Sáez, L.; Bustos, S.; Caro, J.C.; Mualim, J.F. y Pérez, S. (2015). Mercados Públicos de Alimentos en Chile y Recomendaciones para la inclusión de la Agricultura Familiar Campesina. Santiago: INDAP.

Sotomayor, O.; Rodríguez, A.y Rodrigues, M.(2011).Competitividad, sostenibilidade inclusión social en la agricultura. Nuevas direcciones en el diseño de políticas en América Latina y el Caribe. Santiago: CEPAL.

Teo, Carla Rosane Paz Arruda (2018)."The partnership between the Brazilian School Feeding Program and family farming: A way for reducing ultra-processed foods in school meals". Public Health Nutrition, vol. 21, núm.1.

Universidad de Chile (2014). Encuesta Nacional de Consumo Alimentario. Recuperado el 27 de marzo de 2018 de http://web.minsal.cl/sites/default/files/ ENCA-INFORME_FINAL.pdf.

Valencia, V.; Wittman, H. y Blesh, J. (2019). Structuring Markets for Resilient Farming Systems. Agronomy for Sustainable Development, vol. 39, núm 2.

Zoccal Gomes, G. y Esteves, P. (2018). “The BRICS Effect: Impacts of South-South Cooperation in the Social Field of International Development Cooperation". IDS Bulletin, vol. 49, núm. 3 . 
